\title{
A proposal of a standard rainbow false color scale for thermal medical images
}

\author{
by R. Vardasca*** and J. Gabriel*
}

\begin{abstract}
* LABIOMEP, IDMEC-FEUP Campus, Faculty of Engineering, University of Porto, Porto, Portugal, ricardo.vardasca@fe.up.pt, jgabriel@fe.up.pt

${ }^{* *}$ Medical Imaging Research Unit, Faculty of Computing, Engineering and Science, University of

South Wales, Wales, United Kingdom, ricardo.vardasca@southwales.ac.uk
\end{abstract}

\begin{abstract}
Medical thermal imaging offers the opportunity of human body physiology monitoring. The frequent use of false color scales in those images has the objective of being a visual aid for human eye interpretation. However, several scales are being used, which may lead to different subjective interpretations. Is objective of this study to raise the need of uniformity in adoption of an internationally accepted standard false color scale and for that purpose a scale is proposed.
\end{abstract}

\section{Introduction}

Medical thermal imaging is a non-invasive medical screening modality that maps human body surface temperature providing real-time peripheral microcirculation and autonomous system monitoring. A Canadian physician called Ray Lawson has introduced this medical imaging modality into clinical practic in 1956, when he tried to characterize the surface temperature of breast carcinomas It has been applied clinically with success in inflammatory arthritis, osteoarthritis, soft tissue rheumatism, enthesopathies, tennis elbow, fibromyalgia, complex regional pain syndrome, peripheral circulation, fever screening, burns, renal dialysis and malignant diseases [1-2].

The first medical thermal images were in a single color and taken using an evaporograph, although the equipment's evolved, becoming more portable, sensitive and of better quality, the resultant images also had shown significant improvements, especially when computers were introduced and images starting to be digital, it has allowed two types of analysis, the subjective in the screen and the quantitative. The subjective analysis was performed through observing regions of different temperatures that would be more accurate when using a false color scale, in which each color represented a temperature value. The quantitative analysis is made with aid of geometrical regions of interest (ROI, lines, ellipses and rectangles) where measurements are obtained resulting in ROls statistical values (minimum, mean and maximum temperatures and standard deviation) [3].

Despite the thermal images being formed of radiometric data resultant from the thermal radiation value perceived by the thermal sensors of the camera, which are transformed in voltage values and coded in the numeric values according to the pixels resolution and sensors temperature range. The software packages for thermal imaging analysis offer different false color palettes for a better visual understanding, which are able to produce different interpretations. That is due the different perceptions of color among human beings.

The visible spectrum contains numerous colors that are distinguished by wavelength and amplitude; wavelength determines color and amplitude determines brightness. Of these colors, the human eye can distinguish about 10,000. The visible spectrum, however, is often identified by the seven prominent colors we see in the rainbow. In 1666, Isaac Newton named these colors red, orange, yellow, green, blue, indigo, and violet, which are often referred to by the mnemonic acronym ROY G BIV.

More commonly, however, the spectrum is arranged in order of wavelength, shortest to longest, and divided into segments identified as violet (380-450nm), blue (450-490nm), green (490-560nm), yellow (560-590nm), orange (590$630)$, and red (630-780), Fig. 1:

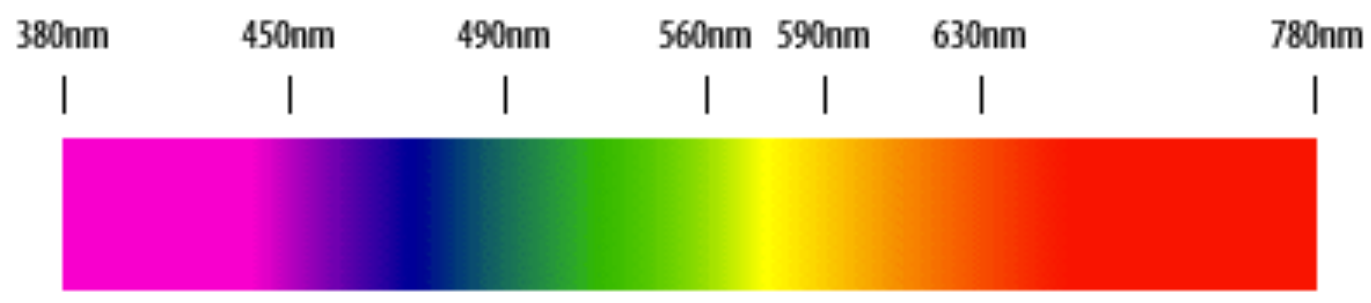

Fig. 1 - The visible spectrum. 
The color perception can be explained by the CIE (Commission Internationale de l'Eclairage - the International Commission on Illumination) system, Fig. 2, which is based on three spectral curves for the CIE primaries. Color is specified by the relative amounts of the CIE primaries $X$ (a linear combination of cone response curves chosen to be nonnegative), $Y$ (luminance) and $Z$ (quasi-equal to blue stimulation or cone response) that match a given color.

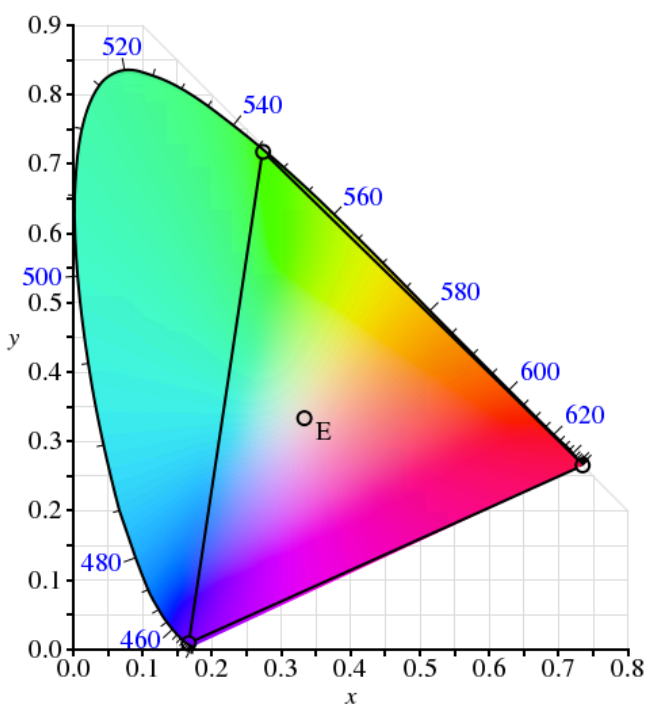

Fig. 2 - The Gamut of the CIE RGB primaries and location of primaries on the CIE 1931 xy chromaticity diagram.

In the 1920s, W. David Wright [4] and John Guild [5] independently conducted a series of experiments on human sight, which laid the foundation for the specification of the CIE XYZ color space. The experiments were conducted by using a circular split screen (a bipartite field) 2 degrees in diameter, which is the angular size of the human fovea. On one side of the field a test color was projected and on the other side, an observer-adjustable color was projected. The adjustable color was a mixture of three primary colors, each with fixed chromaticity, but with adjustable brightness.

Although Wright and Guild's experiments were carried out using various primaries at various intensities, and although they used a number of different observers, all of their results were summarized by the standardized CIE RGB color matching functions $r(\lambda), g(\lambda)$ and $b(\lambda)$, obtained using three monochromatic primaries at standardized wavelengths of $700 \mathrm{~nm}$ (red), $546.1 \mathrm{~nm}$ (green) and $435.8 \mathrm{~nm}$ (blue). The color matching functions are the amounts of primaries needed to match the monochromatic test primary. These functions are shown in Fig. 3. It can be observed that $r(\lambda)$ and $g(\lambda)$ are zero at 435.8, $r(\lambda)$ and $b(\lambda)$ are zero at 546.1 and $g(\lambda)$ and $b(\lambda)$ are zero at $700 \mathrm{~nm}$, since in these cases the test color is one of the primaries. The primaries with wavelengths $546.1 \mathrm{~nm}$ and $435.8 \mathrm{~nm}$ were chosen because they are easily reproducible monochromatic lines of a mercury vapor discharge. The $700 \mathrm{~nm}$ wavelength, which in 1931 was difficult to reproduce as a monochromatic beam, was chosen because the eye's perception of color is rather unchanging at this wavelength, and therefore small errors in wavelength of this primary would have little effect on the results.

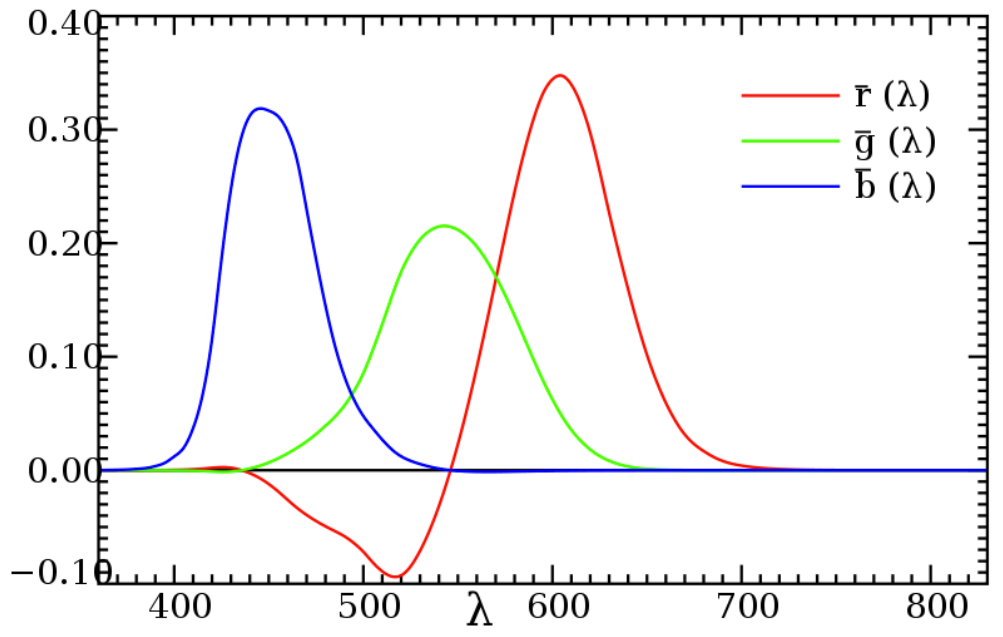

Fig. 3 - The CIE 1931 RGB Color matching functions. 
However, recent studies [6] have shown that the CIE does not represent the human eye perception of color, there are even difference between genders (Fig. 4), bringing to discussion the proposed standard rainbow scale proposed by Borland [7] that is widely implemented in software's that use false color maps over radiometric images.
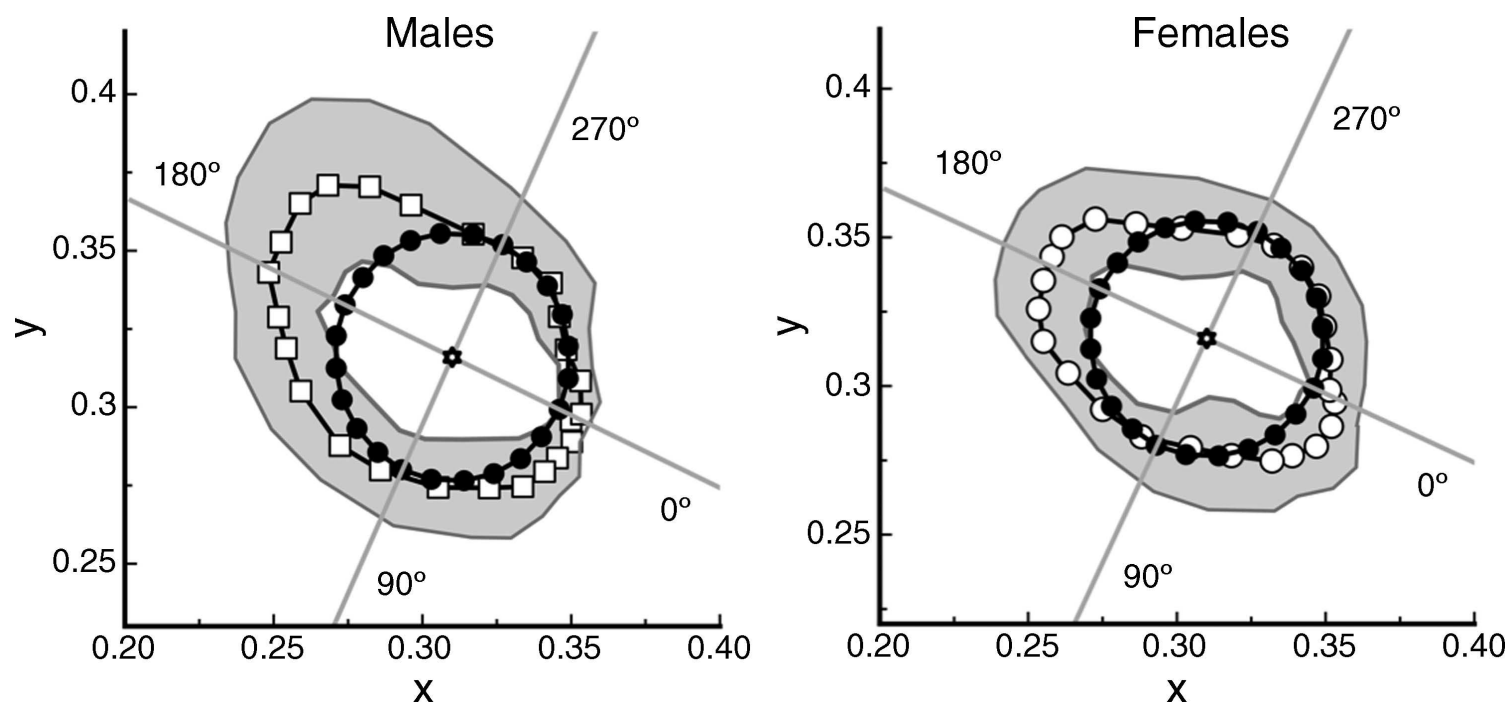

Fig. 3 - (Left) Male and (right) female color matches in CIE 1931 xy color space. The black spots are the probe chromaticities and the open symbols (squares for males and circles for females) are the averaged matched chromaticities. The dark gray curves and the gray shaded areas are $\pm 1 \mathrm{SD}$. The light gray lines are the cardinal axes. The open star is the background (illuminant $\mathrm{C}$ ), [6].

There is aim of this research to evaluate the use of different false color maps in radiometric thermal images and bring up the discussion in the need of normalization of a false rainbow color map to be used in medical thermal imaging. Proposing an adaptation of the existing rainbow false color map that was defined [7].

\section{Methodology}

A set of IR medical images of different regions of the body containing temperature measurements in grayscale values is used. These images are loaded into MATLAB prototyping software and a range of different false color scales are applied and its differences in subjective interpretation evaluated (Fig. 5). 20 Health professional graded the resultant images from applying the false color maps and the marking scheme used was 12 for best false color map and 1 for the worst.
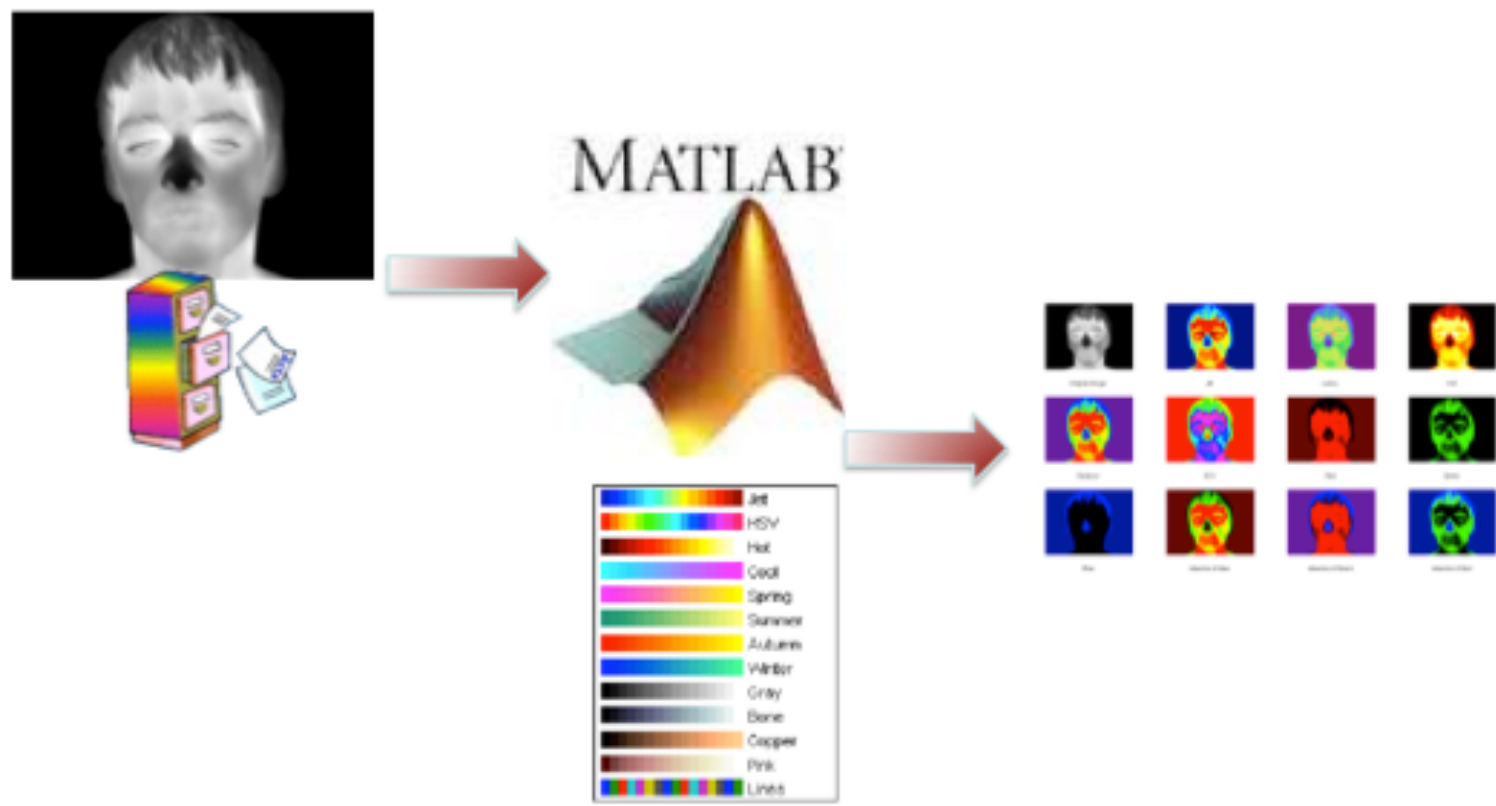

Fig. 5 - Diagram of the methodology used in this experiment. 
The change in the previously defined rainbow scale was based in the average between the mean perception of males and females and based in the CIE model of 1931. Being the $x$ and $y$ adjust by the value of 1.33 in the following equations:

$$
\begin{aligned}
& x=\frac{X}{X+Y+Z} * 1.33 \\
& y=\frac{Y}{X+Y+Z} * 1.33
\end{aligned}
$$

\section{Results}

An example of the obtained comparison of the different false color scales is presented in Fig. 6 , the developed new rainbow false color map is defined as 'Rainbow'. The results were evaluated subjectively by 20 health professionals that deal everyday with medical imaging and from their assessment the preferred scale was the grayscale, but when question which false color scale they preferred, their grading are shown in table 1.

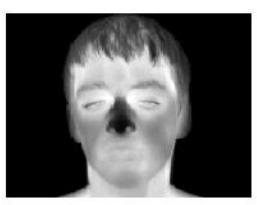

Original Image

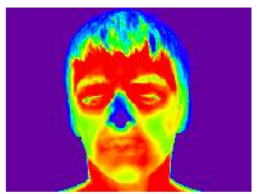

Rainbow

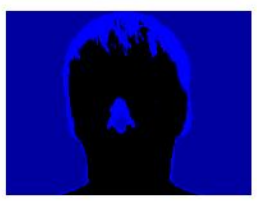

Blue

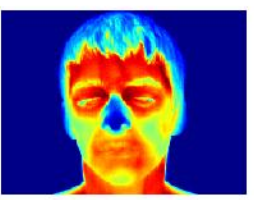

Jet

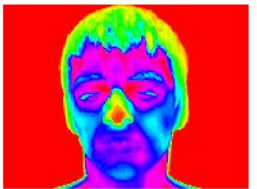

HSY

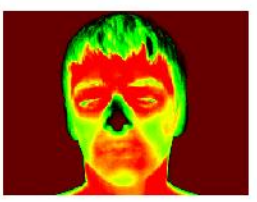

Absence of blue

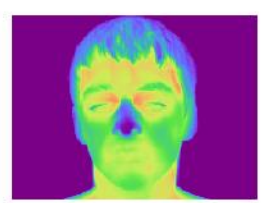

cubicL

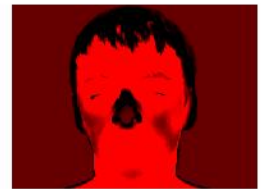

Red

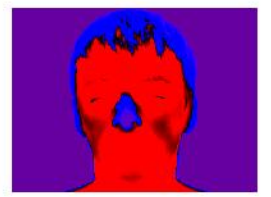

Absence of Green

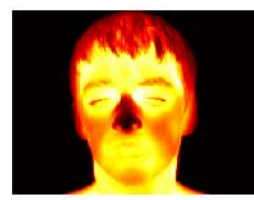

Hot

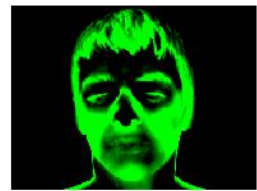

Green

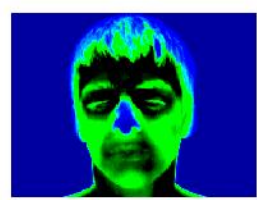

Absence of Red

Fig. 6 - An example of the output of the analysis of different false color scale representation of an IR image.

Table 1. Results from the assessment of the 20 health professionals.

\begin{tabular}{|l|c|}
\hline \multicolumn{1}{|c|}{ Color map } & Votes \\
\hline Original image & 60 \\
\hline Jet & 220 \\
\hline cubicL & 160 \\
\hline Hot & 75 \\
\hline Rainbow & 240 \\
\hline HSV & 120 \\
\hline Red & 20 \\
\hline Green & 35 \\
\hline Blue & 20 \\
\hline Absence of Blue & 40 \\
\hline Absence of Green & 20 \\
\hline Absence of Red & 30 \\
\hline
\end{tabular}




\section{Discussion}

The false color scale with higher perception is the developed 'Rainbow', which has presented a significant result when compared with the conventional rainbow palette previously defined [7]. The new palette facilitates the perception, would improve understanding and facilitates medical images interpretation. A standard is needed, not just in medical thermography but in also the other medical imaging modalities.

\section{Conclusion}

The adoption of the proposed false color scale will enforce the standardization of the analysis of medical IR images, not only thermography uses them, in X-Ray and ultrasound the usage of false color maps is becoming more popular. A further study in false color perception is needed to improve the subjective analysis of thermograms and this proposed change in the conventional rainbow false color map need further investigation.

\section{ACKNOWLEDGMENT}

This research is sponsored by FEDER funds through the program COMPETE - Programa Operacional Factores de Competitividade and by national funds through FCT-Fundação para a Ciência e a Tecnologia, under the Project "AAL4ALL - Padrão de Cuidados Primários para Serviços de AAL" (FCOMP-01-0124-FEDER-13852) co-financed by FEDER, Programa Operacional Factores de Competitividade.

\section{REFERENCES}

[1] Ring E.F.J., Ammer K., "Infrared thermal imaging in medicine". Physiological measurement, vol. 33.3, pp. R33, 2012.

[2] Vardasca R., Simoes R., "Current Issues in Medical Thermography", in Topics in Medical Image Processing and Computational Vision, Tavares J. M. R. S. and Jorge R. M. N. (Eds.), Springer Netherlands, pp. 223-237, 2013.

[3] Ring E.F.J., Jones B.F., "Historical Development of Thermometry and Thermal Imaging in Medicine", Medical Infrared Imaging: Principles and Practices, M. Diakides, J. D. Bronzino and D. R. Peterson (Eds.), Chapter 2, Taylor and Francis / CRC Press, Boca Raton, USA, 2013.

[4] Wright W. D., "A re-determination of the trichromatic coefficients of the spectral colours". Transactions of the Optical Society, vol. 30(4), pp. 141-164, 1928.

[5] Guild J., "The colorimetric properties of the spectrum". Philosophical Transactions of the Royal Society of London. Series A, Containing Papers of a Mathematical or Physical Character, vol. 230, pp. 149-187, 1932.

[6] Murray I.J., Parry N. R., McKeefry D. J., Panorgias, A. "Sex-related differences in peripheral human color vision: A color matching study". Journal of vision, vol. 12(1), pp.18, 2012.

[7] Borland D., Taylor R.M.II, "Rainbow Color Map (Still) Considered Harmful", IEEE Computer Graphics and Applications, Volume 27, pp. 14-17, 2007. 